\title{
1 Effect of reaction time and algae loading on water-soluble and 2 insoluble biocrude fractions from hydrothermal liquefaction of \\ 3 \\ algae
}

4

10

\author{
Donghai Xu $\mathrm{u}^{\mathrm{a}, \mathrm{b}}$, Phillip E. Savage $\mathrm{e}^{\mathrm{b}, \mathrm{c}, *}$
}

a. Key Laboratory of Thermo-Fluid Science \& Engineering, Ministry of Education, School of Energy and Power Engineering, Xi'an Jiaotong University, Xi'an, Shaanxi Province 710049, China

b. Department of Chemical Engineering, University of Michigan, Ann Arbor, MI, 48109, United States

c. Department of Chemical Engineering, Pennsylvania State University, University Park, PA, 16802, United States

1 ABSTRACT: Hydrothermal liquefaction (HTL) of algae produces a water-insoluble organic phase (biocrude) and an aqueous phase that also contains algae-derived organic compounds (water-soluble biocrude). We determined the influences of the HTL reaction time and algae loading (wt $\%$ biomass in algae paste) on the yields and compositions of these two biocrudes. To the best of our knowledge this is the first detailed study of the water-soluble biocrude and how its yield and composition vary with processing conditions. Increasing the HTL reaction time from 10 to 60 min led to lower yields of water-soluble biocrude (from 6.4 to $3.6 \mathrm{wt} \%$ ) and higher yields of water-insoluble biocrude (from 38 to $41 \mathrm{wt} \%$ ). The total biocrude yield, however, was insensitive to the processing time. Longer HTL processing times also led to fewer and lighter GC-elutable compounds in the water-soluble biocrude. Increasing the algae loading from 8.6 to $18.7 \mathrm{wt} \%$ led to higher yields of water-insoluble biocrude (from

\footnotetext{
*Corresponding author. Tel.:+1 814867 5876; fax: +1 814-865-7846.

E-mail address: psavage@psu.edu (P.E. Savage).
} 
2129 to $44 \mathrm{wt} \%$ ) and lower yields of water-soluble biocrude (from 6.6 to $2.6 \mathrm{wt} \%$ ). The increase in the

22 water-insoluble portion was much greater than the decrease in the water-soluble portion, so the total

23 biocrude yield increased with loading. Increasing the wt $\%$ of algae in the slurry placed in the reactors

24 also produced biocrudes with greater heating value. HTL of algae paste with higher biomass content had

25 the twin benefits of providing more total biocrude and a higher quality biocrude. Reaction time and

26 algae loading can be used to manipulate the proportions of water-soluble biocrude and biocrude that

27 spontaneously separates from the aqueous phase after HTL.

28 Keywords: algae, hydrothermal liquefaction, biocrude, biorefinery

\section{1. Introduction}

Hydrothermal liquefaction (HTL) converts wet organic material (e.g., biomass) into energy-dense gasification or pyrolysis, that require the biomass to be dried [3]. HTL of wet algal biomass produces an organic biocrude fraction, an aqueous fraction containing numerous polar organic compounds [4], a gas fraction, and a solid residue. The latter two product fractions generally appear in yields of just a few percent.

37 Two different approaches can be used to recover the biocrude from HTL. One approach is to allow the biocrude and aqueous phases to separate spontaneously due to their immiscibility and density difference 
42 [e.g.,7]. The former approach has the advantage of being a simpler and "greener" process as no solvent

43 extraction step is needed and no organic solvent is required. The latter approach has the advantage of

44 producing a modestly higher biocrude yield, since water-soluble material is extracted and recovered in

45 the biocrude fraction. These water-soluble extractables tend to be richer in heteroatoms than the

46 water-insoluble components of the biocrude [8], so this solvent extraction approach tends to produce a

47 biocrude of lower quality (less heating value, more heteroatoms) than the former approach.

48 More generally, whether or not the water-soluble materials are extracted as biocrude affects aspects of

49 the biorefinery apart from considerations of biocrude quantity and quality. If the DCM-soluble

50 components are removed from the aqueous phase, then that aqueous phase will have a lower

51 concentration of organic compounds. This lower loading could be beneficial if the aqueous phase is

52 recycled directly to algae growth ponds. The reduced organic content would provide a less hospitable

53 environment for growth of undesired invasive heterotrophic organisms in the algae pond. The lower

54 concentration could be a disadvantage, however, if the biorefinery employed a process such as catalytic

55 hydrothermal gasification to convert the dissolved aqueous-phase organic compounds into fuel gases

56 (e.g., $\mathrm{CH}_{4}$ ) to provide heat and power for the biorefinery [9]. Removing the DCM-soluble material from

57 the aqueous phase would also hinder recovery of nitrogen and its recycling for algae growth, as more of

58 the $\mathrm{N}$ atoms would be removed from the aqueous phase and partitioned into the biocrude fraction.

59 The discussion above shows that the decision about whether to use an organic solvent for biocrude

60 extraction or whether to rely solely on spontaneous phase separation of the HTL reactor effluent affects

61 several aspects of biorefinery operation, economic viability, and environmental sustainability. To make

62 this decision, one desires to know how solvent extraction affects the quantity and composition of the 

biocrude and the composition of the aqueous phase coproduct. To the best of our knowledge, our earlier preliminary work on this topic remains the only account in the archival scientific literature [8]. That account compared the biocrudes and aqueous phases from HTL at a single reaction condition and recovered by the two different approaches. The present study expands upon that work by determining

67 the influence of the HTL conditions on the characteristics of the biocrude that spontaneously separates

68 from the aqueous phase post reaction and the biocrude that can be extracted from the aqueous phase

69 using DCM. This information will be useful in deciding how best to design and operate an algal

70 biorefinery that employs HTL.

\section{2. Experimental section}

\section{2.1. Experimental procedures}

73 We used the preservative-free Nannochloropsis sp. slurry, reactors, reagents, etc., that have been fully

74 described in our previous report [8]. HTL experiments were carried out at $350{ }^{\circ} \mathrm{C}$ under two sets of

75 conditions. One set explored HTL at four different batch holding times (10, 30, 45, and 60 min) and a

76 fixed algae loading of $14.1 \mathrm{wt} \%$. The other set explored HTL at four different algae loadings (8.6, 11.4,

7716.5 , and $18.7 \mathrm{wt} \%$ ) at a fixed batch holding time of $20 \mathrm{~min}$.

78 We loaded the reactor with known amounts of algae slurry and deionized water, sealed the reactor,

79 and then placed it in a Techne Fluidized Sand Bath (Model SBL-2) maintained at $350{ }^{\circ} \mathrm{C}$. When the

80 desired reaction time was reached, we removed the reactor from the sand bath and quenched it in an 

before collecting products.

83 We followed the procedure denoted as method 2 (M2) in our previous article [8] to collect, separate,

84 and quantify the biocrude. Briefly, we opened the reactor and directly collected the aqueous phase from

85 the cool, equilibrated reactor by a pipet. We then added DCM to the reactor to dissolve and collect the

86 water-insoluble biocrude (B) in the reactor as well as that adhering to the pipet. Note that the DCM did

87 not come into contact with the aqueous phase co-product. DCM was added in these experiments for

88 convenience and to ensure complete recovery of the water-insoluble biocrude. Its addition would likely

89 be optional in a large-scale biorefinery where the biocrude-water separation could be done continuously

90 and at higher temperatures. Evaporating the DCM by flowing $\mathrm{N}_{2}$ in a drying apparatus (Vertex ${ }^{\mathrm{TM}}$

91 EVAPORATOR) led to isolation of the water-insoluble biocrude.

92 Centrifugation separated the aqueous phase that had been collected from any accompanying solids,

93 which were subsequently dried. We added DCM to the solids-free aqueous phase and mixed it

94 thoroughly to extract the DCM-soluble, water-soluble material. These DCM-soluble and water-soluble

95 compounds constitute the aqueous-phase contribution to the biocrude $\left(\mathrm{B}_{\mathrm{A}}\right)$. The total mass of the

96 biocrude $\left(\mathrm{B}_{\mathrm{T}}\right)$ is the sum of the masses of the water-insoluble $(\mathrm{B})$ and water-soluble $\left(\mathrm{B}_{\mathrm{A}}\right)$ components.

97 We further separated each biocrude into light (hexane-soluble) and heavy (hexane-insoluble) fractions

98 as described previously [10].

Biocrude samples were shipped to Atlantic Microlab, Inc., for elemental analysis. We identified 
components of the biocrudes by redissolving them in DCM and analyzing by GC-MS. Nuclear magnetic resonance (NMR) and Fourier transform infrared spectroscopic analysis (FTIR) of biocrudes were also conducted to characterize the biocrude. Detailed information about these instruments and procedures was reported previously [8].

The higher heating value (HHV) of biocrude was estimated by using the Dulong formula,

$\mathrm{HHV}(\mathrm{MJ} / \mathrm{kg})=0.338 \mathrm{C}+1.428(\mathrm{H}-\mathrm{O} / 8)+0.095 \mathrm{~S}$

where $\mathrm{C}, \mathrm{H}, \mathrm{O}$, and $\mathrm{S}$ are the wt $\%$ of each element in the biocrude.

Additionally, the biocrude yield, the fraction of total biocrude from the aqueous phase, the fraction of light biocrude, and the energy recovery in the biocrude were calculated as follows:

Biocrude yield $=\frac{\text { Mass of biocrude }}{\text { Mass of algae in reactor }} 100 \%$

Fraction of total biocrude from aqueous phase $=\frac{\mathrm{B}_{\mathrm{A}}}{\mathrm{B}+\mathrm{B}_{\mathrm{A}}} \times 100 \%$

Fraction of light biocrude $=\left(1-\frac{\text { Mass of measured } \mathrm{B}^{\mathrm{H}}}{\text { Mass of } \mathrm{B}}\right) \times 100 \%$

Energy recovery of biocrude $=\frac{\text { HHV of biocrude } \times \text { Mass of the biocrude }}{\text { HHV of initial dry algae } \times \text { Mass of the algae feedstock }} \times 100 \%$

Three independent runs were carried out at the same conditions to determine the uncertainties in the experimental data. Results reported herein are average values and their uncertainties are the sample standard deviations.

\section{Results and discussions}

The section provides results about the biocrude yields and compositions. To the extent possible, the discussion focuses on examining the effects of time and algae loading on the water-soluble biocrude fraction and on characterizing the light and heavy fractions of the water-soluble biocrude, as this report 
121 is the first to consider these topics. The mass balances in the experiments ranged from 90-95 wt\%.

122 3.1. Biocrude yields

123 Table 1 shows the influence of reaction time on biocrude yields. The yield of water-soluble biocrude

$124\left(\mathrm{~B}_{\mathrm{A}}\right)$ declined from 6.4 to $3.6 \mathrm{wt} \%$ as time increased, but the yield of water-insoluble biocrude (B) rose

125 from 38.2 to $40.5 \mathrm{wt} \%$ as time increased from $10-60 \mathrm{~min}$. Accordingly, the total biocrude yield $\left(\mathrm{B}_{\mathrm{T}}\right)$ was

126 time-invariant at about $44 \mathrm{wt} \%$. Table 1 also shows that the fraction of total biocrude from the aqueous

127 phase decreased from 14.4 to $8.1 \mathrm{wt} \%$ as time increased from 10-60 min. Accordingly, one expects the

128 total biocrude collected at short times to be richer in $\mathrm{O}$ and $\mathrm{N}$ (more water-soluble compounds) than the

129 biocrude collected at longer times. The absence of much variation in the total biocrude yield with time at

$130350{ }^{\circ} \mathrm{C}$ has been observed previously [10]. That the relative contribution of the aqueous phase biocrude

131 to the total amount of biocrude changes with HTL reaction time has not been previously documented.

Table 1 Biocrude yields (wt $\%$ ) from HTL at $350{ }^{\circ} \mathrm{C}$ and different times (14.1 wt $\%$ algae loading).

\begin{tabular}{|c|c|c|c|c|}
\hline Time (min) & 10 & 30 & 45 & 60 \\
\hline Yield of water-insoluble biocrude (B) & $38.2 \pm 1.4$ & $39.2 \pm 1.3$ & $40.1 \pm 1.3$ & $40.5 \pm 1.5$ \\
\hline Yield of water-soluble biocrude $\left(\mathrm{B}_{\mathrm{A}}\right)$ & $6.4 \pm 0.5$ & $4.5 \pm 0.6$ & $4.6 \pm 0.5$ & $3.6 \pm 0.4$ \\
\hline Total biocrude yield & $44.6 \pm 1.9$ & $43.7 \pm 2.0$ & $44.7 \pm 1.8$ & $44.1 \pm 1.9$ \\
\hline \multicolumn{5}{|c|}{ Fraction of total biocrude from aqueous phase } \\
\hline & $14.4 \pm 1.6$ & $9.3 \pm 0.8$ & $10.2 \pm 0.8$ & $8.1 \pm 0.7$ \\
\hline$(\mathrm{wt} \%)$ & & & & \\
\hline
\end{tabular}

133 Table 2 shows that the yield of water-soluble biocrude $\left(B_{A}\right)$ first increased and then decreased from

1346.6 to $3.0 \mathrm{wt} \%$, as algae loading increased from 8.6 to $18.7 \mathrm{wt} \%$. In contrast, the yields of 
water-insoluble and total biocrude increased from 29.2 to $44.0 \mathrm{wt} \%$ and from 34.8 to $47.0 \mathrm{wt} \%$,

respectively, as the algae loading increased. The fraction of the total biocrude that was in the aqueous yield from 36 to $46 \mathrm{wt} \%$ [10].

Table 2 Biocrude yields (wt $\%$ ) from HTL at $350{ }^{\circ} \mathrm{C}$ and different algae loadings (20 min).

\begin{tabular}{|c|c|c|c|c|}
\hline Algae loading (wt $\%$ ) & 8.6 & 11.4 & 16.5 & 18.7 \\
\hline Yield of water-insoluble biocrude (B) & $29.2 \pm 2.1$ & $38.1 \pm 1.8$ & $41.1 \pm 1.5$ & $44.0 \pm 2.4$ \\
\hline Yield of water-soluble biocrude $\left(\mathrm{B}_{\mathrm{A}}\right)$ & $5.6 \pm 0.5$ & $6.6 \pm 0.6$ & $3.7 \pm 0.5$ & $3.0 \pm 0.4$ \\
\hline Total biocrude yield & $34.8 \pm 2.6$ & $44.7 \pm 2.4$ & $44.7 \pm 2.0$ & $47.0 \pm 2.9$ \\
\hline \multicolumn{5}{|c|}{ Fraction of total biocrude from aqueous phase } \\
\hline & $16.1 \pm 1.4$ & $14.7 \pm 1.2$ & $8.2 \pm 0.8$ & $6.4 \pm 0.4$ \\
\hline$(\mathrm{wt} \%)$ & & & & \\
\hline
\end{tabular}

142 Overall, both increased time and increased algae loading decreased the yield of water-soluble

143 biocrude. This decrease likely occurred because some of the molecules initially formed from algae HTL

144 contain polar groups and hence enjoy solubility in water. As the reaction progressed, however, such

145 compounds could undergo deamination, decarboxylation, dehydration, and oligomerization. One

146 expects the oligomerization pathways, which involve bimolecular reactions, to become more favorable

147 as the algae loading increases. The net effect is that the molecules became larger and less polar, which

148 rendered them less soluble in water. 
149 The results show that reaction time and algae loading can be used to control the proportions of 150 water-soluble biocrude and biocrude that spontaneously separates from the aqueous phase after HTL.

151 Such control could be useful, for example, to ensure that the aqueous phase retains sufficient organic 152 content for a catalytic hydrothermal gasification unit operation to generate enough fuel gas to meet the 153 heat and power demands of the biorefinery.

\subsection{Fraction of light biocrude}

Fig. 1 illustrates how the portion of the biocrudes that falls into the light (hexane-soluble) fraction changed with time and algae loading. This metric is a proxy for biocrude quality, as the hexane-soluble compounds would be less polar and of moderate boiling point and thus more desirable for fuel use than would the heavier, more polar, hexane-insoluble compounds. The fractions of the water-insoluble and water-soluble biocrudes that were hexane-soluble increased from 62 to $77 \mathrm{wt} \%$ and from 67 to $78 \mathrm{wt} \%$, respectively, as time increased from 10 to $60 \mathrm{~min}$. Recall that the fraction of the total biocrude that was water-soluble decreased from 14 to $8 \%$ over this same time range. It seems that the same reactions that rendered less of the biocrude to be water-soluble also rendered more of it hexane-soluble. That is, the reactions that made the molecules less polar both reduced the solubility in water and increased the solubility in hexane.

As algae loading increased from 8.6 to $18.7 \mathrm{wt} \%$, the fraction of light (hexane-soluble) material in the water-insoluble biocrude (B) first increased and then decreased, but that in the water-soluble biocrude $\left(\mathrm{B}_{\mathrm{A}}\right)$ decreased monotonically from 77 to $51 \mathrm{wt} \%$. Recall that the yield of water-soluble biocrude was lower at the higher algae loadings. This trend, along with that noted here for the light biocrude, indicates 
a)

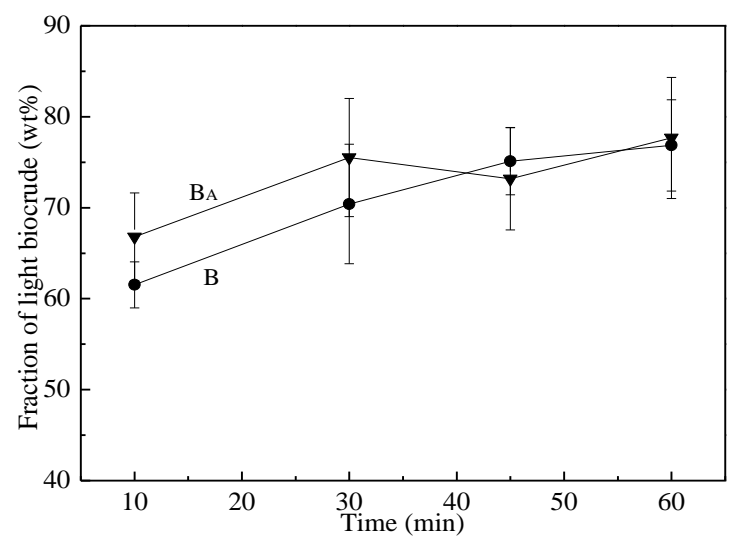

b)

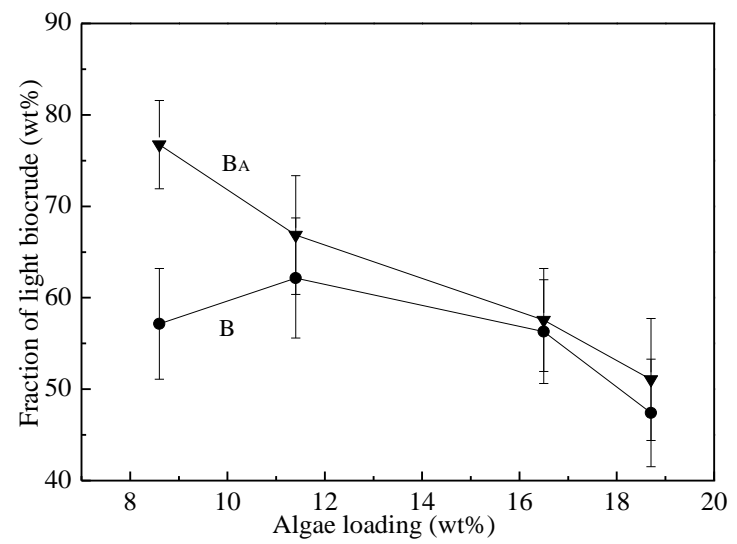

174

175

Fig. 1. Fraction of light biocrude in $B$ and $B_{A}$ from $H T L$ at $350{ }^{\circ} \mathrm{C}$ : a) different times, 14.1 wt $\%$ algae loading, and $b$ )

different algae loadings, 20 min.

\subsection{Elemental compositions and higher heating values}

The yields of light, heavy, and total biocrude are important metrics for assessing HTL, as they relate

the amount of product obtained. The compositions of these biocrudes are also very significant, however,

as this metric relates to the quality of the product obtained. Biocrudes with more $\mathrm{H}$ and $\mathrm{C}$ and less $\mathrm{O}, \mathrm{N}$, and $\mathrm{S}$ are more desirable products.

Table 3 lists the elemental compositions and estimated higher heating values (HHV) of the water-soluble and water-insoluble biocrudes and their heavy (hexane-insoluble) fractions formed at different HTL reaction times. The $\mathrm{O}$ content was calculated by difference from the experimental $\mathrm{C}, \mathrm{H}, \mathrm{N}$, and $\mathrm{S}$ values and reserving $1 \mathrm{wt} \%$ for other elements not assessed. 
186 (hexane-insoluble) components $\left(\mathrm{B}^{\mathrm{H}}, \mathrm{B}_{\mathrm{A}}{ }^{\mathrm{H}}\right)$ produced at different HTL reaction times $\left(350{ }^{\circ} \mathrm{C}, 14.1 \mathrm{wt} \%\right.$ algae loading).

\begin{tabular}{|c|c|c|c|c|c|c|c|c|c|}
\hline & Time & $\mathrm{C}$ & $\mathrm{H}$ & $\mathrm{N}$ & $\mathrm{S}$ & $\mathrm{O}$ & $\mathrm{N} / \mathrm{C}$ & $\mathrm{O} / \mathrm{C}$ & HHV \\
\hline \multicolumn{10}{|l|}{ Item } \\
\hline & (min) & $(\mathrm{wt} \%)$ & $(w t \%)$ & $(w t \%)$ & $(\mathrm{wt} \%)$ & $(\mathrm{wt} \%)$ & - & - & $(\mathrm{MJ} / \mathrm{kg})$ \\
\hline Dry algae & - & 52.6 & 7.35 & 8.58 & 0.57 & 27.0 & 0.152 & 0.477 & 24.8 \\
\hline \multirow{4}{*}{ B } & 10 & 77.5 & 10.0 & 4.85 & 0.56 & 7.08 & 0.063 & 0.091 & 39.3 \\
\hline & 30 & 77.5 & 10.0 & 4.60 & 0.48 & 7.36 & 0.059 & 0.095 & 39.3 \\
\hline & 45 & 78.3 & 10.0 & 4.54 & 0.48 & 6.64 & 0.058 & 0.085 & 39.6 \\
\hline & 60 & 78.1 & 10.0 & 4.48 & 0.50 & 6.96 & 0.057 & 0.089 & 39.5 \\
\hline \multirow{4}{*}{$\mathrm{B}_{\mathrm{A}}$} & 10 & 63.4 & 8.76 & 10.2 & 0.20 & 17.5 & 0.161 & 0.276 & 30.8 \\
\hline & 30 & 62.6 & 8.72 & 10.7 & 0.10 & 17.8 & 0.171 & 0.285 & 30.4 \\
\hline & 45 & 64.4 & 9.07 & 10.0 & 0.20 & 16.3 & 0.155 & 0.253 & 31.8 \\
\hline & 60 & 63.1 & 8.88 & 10.4 & 0.16 & 17.5 & 0.164 & 0.277 & 30.9 \\
\hline \multirow{4}{*}{$\mathrm{B}^{\mathrm{H}}$} & 10 & 72.6 & 8.49 & 6.11 & 0.63 & 12.2 & 0.084 & 0.168 & 34.5 \\
\hline & 30 & 74.2 & 8.60 & 6.16 & 0.64 & 10.4 & 0.083 & 0.141 & 35.5 \\
\hline & 45 & 74.5 & 8.53 & 5.86 & 0.58 & 10.5 & 0.079 & 0.141 & 35.6 \\
\hline & 60 & 75.8 & 8.66 & 5.91 & 0.52 & 9.09 & 0.078 & 0.120 & 36.4 \\
\hline \multirow{3}{*}{$\mathrm{B}_{\mathrm{A}}{ }^{\mathrm{H}}$} & 10 & 63.0 & 8.04 & 11.1 & 0.72 & 17.1 & 0.176 & 0.271 & 29.8 \\
\hline & 30 & 63.5 & 7.72 & 9.52 & 1.18 & 18.1 & 0.150 & 0.284 & 29.4 \\
\hline & 45 & 63.7 & 7.81 & 9.98 & 1.19 & 17.3 & 0.157 & 0.271 & 29.7 \\
\hline
\end{tabular}




$\begin{array}{lllllllll}60 & 63.8 & 7.62 & 9.77 & 1.17 & 17.6 & 0.153 & 0.276 & 29.4\end{array}$

187 Our previous article [8] discussed the differences between the water-soluble and water-insoluble

188 biocrudes, so here we focus on the effect of HTL reaction time on the different biocrudes. To the best of

189 our knowledge, Table 3 presents the first data for the elemental composition of the heavy fraction of the

190 water-soluble biocrude from HTL.

191 The HTL processing time seemed to have little effect on the composition of the biocrude (B) that

192 spontaneously separated post-liquefaction or on the water-soluble biocrude component $\left(\mathrm{B}_{\mathrm{A}}\right)$. There are

193 modest differences, but we believe these can be attributed to run-to-run variation in the experimental

194 results. The composition of the heavy fractions of these two biocrudes did show some trends with

195 reaction time, however. The heavy fraction of the water-insoluble biocrude $\left(\mathrm{B}^{\mathrm{H}}\right)$ showed a modestly

196 increasing carbon content and heating value and a decreasing oxygen content as time increased. The

197 heavy fraction of the water-soluble biocrude showed the highest nitrogen content at the shortest time.

198 Table 4 displays the elemental compositions and HHVs of the biocrudes and their heavy fractions

199 formed at different algae loadings. The carbon content of both water-soluble and water-insoluble

200 biocrudes was generally higher when produced at higher algae loadings. The oxygen contents tended to

201 be lower, so the heating value of the biocrudes tended to be greater at the higher algae loadings. Thus,

202 performing HTL at the higher algae loadings has the twin benefits of producing higher yields of

203 biocrude and producing biocrude with a higher energy density. We note that Peterson et al. [1], on the

204 basis of process engineering considerations, suggested a target algae loading of $15-20 \mathrm{wt} \%$ for HTL.

Table 4 Elemental composition and HHV of water-insoluble (B) and water-soluble $\left(\mathrm{B}_{\mathrm{A}}\right)$ biocrudes and their heavy 


\begin{tabular}{|c|c|c|c|c|c|c|c|c|c|}
\hline \multirow{2}{*}{ Item } & Algae loading & $\mathrm{C}$ & $\mathrm{H}$ & $\mathrm{N}$ & S & $\mathrm{O}$ & $\mathrm{N} / \mathrm{C}$ & $\mathrm{O} / \mathrm{C}$ & HHV \\
\hline & $(\mathrm{wt} \%)$ & $(\mathrm{wt} \%)$ & $(\mathrm{wt} \%)$ & $(\mathrm{wt} \%)$ & $(\mathrm{wt} \%)$ & $(\mathrm{wt} \%)$ & - & - & $(\mathrm{MJ} / \mathrm{kg})$ \\
\hline \multirow{4}{*}{ B } & 8.6 & 76.8 & 10.0 & 4.44 & 0.62 & 8.11 & 0.058 & 0.106 & 38.9 \\
\hline & 11.4 & 76.4 & 10.1 & 4.64 & 0.59 & 8.33 & 0.061 & 0.109 & 38.7 \\
\hline & 16.5 & 77.4 & 10.3 & 4.64 & 0.45 & 7.22 & 0.060 & 0.093 & 39.6 \\
\hline & 18.7 & 77.7 & 10.2 & 4.81 & 0.39 & 6.90 & 0.062 & 0.089 & 39.6 \\
\hline \multirow{4}{*}{$\mathrm{B}_{\mathrm{A}}$} & 8.6 & 63.5 & 8.38 & 10.9 & 0.28 & 16.9 & 0.172 & 0.266 & 30.4 \\
\hline & 11.4 & 63.7 & 8.28 & 11.2 & 0.31 & 16.5 & 0.176 & 0.260 & 30.4 \\
\hline & 16.5 & 68.2 & 8.69 & 9.94 & 0.32 & 12.9 & 0.146 & 0.189 & 33.2 \\
\hline & 18.7 & 65.2 & 8.74 & 10.8 & 0.34 & 15.0 & 0.165 & 0.230 & 31.9 \\
\hline \multirow{4}{*}{$\mathrm{B}^{\mathrm{H}}$} & 8.6 & 74.5 & 8.77 & 6.11 & 0.73 & 9.92 & 0.082 & 0.133 & 36.0 \\
\hline & 11.4 & 74.7 & 8.92 & 6.20 & 0.72 & 9.42 & 0.083 & 0.126 & 36.4 \\
\hline & 16.5 & 76.7 & 9.13 & 6.07 & 0.46 & 7.63 & 0.079 & 0.099 & 37.6 \\
\hline & 18.7 & 76.9 & 9.04 & 5.91 & 0.37 & 7.76 & 0.077 & 0.101 & 37.6 \\
\hline \multirow{4}{*}{$\mathrm{B}_{\mathrm{A}}{ }^{\mathrm{H}}$} & 8.6 & 63.1 & 8.05 & 10.8 & 0.79 & 17.3 & 0.170 & 0.274 & 29.8 \\
\hline & 11.4 & 61.0 & 8.25 & 10.3 & 0.83 & 19.6 & 0.169 & 0.321 & 29.0 \\
\hline & 16.5 & 63.8 & 8.26 & 10.6 & 0.79 & 16.5 & 0.167 & 0.259 & 30.5 \\
\hline & 18.7 & 63.2 & 8.19 & 10.3 & 0.91 & 17.4 & 0.163 & 0.275 & 30.0 \\
\hline
\end{tabular}

207 The influence of algae loading on the heavy fraction of the water-insoluble biocrude $\left(\mathrm{B}^{\mathrm{H}}\right)$ was 208 essentially the same as its influence on the whole biocrude. The carbon content and heating value were 
209 higher at the higher loadings and the oxygen and sulfur contents were lower at the higher loadings. The

210 algae loading seemingly had no discernible effect on the compositions of the heavy fraction of the 211 water-soluble biocrude.

212 Table 5 shows the masses, energy densities, and energy recoveries for both biocrudes at two HTL

213 conditions. The biocrudes that spontaneously separates from the aqueous phase post-HTL (B) contained

214 about $60 \%$ of the chemical energy in the original algal biomass. The "heavy" fractions of those

215 biocrudes $\left(\mathrm{B}^{\mathrm{H}}\right)$ contained about $40 \%$ of the chemical energy in those biocrudes. The biocrudes extracted

216 from the aqueous phase $\left(B_{A}\right)$ contained $5-8 \%$ of the chemical energy in the biomass. By performing the

217 DCM extraction of the aqueous phase, this material can be recovered as biocrude and potentially refined

218 into liquid fuels. This extraction step, however, removes material from the aqueous phase that could be

219 converted to fuel gases for heat and power in the biorefinery. The total energy recoveries under these

220 two HTL conditions were $65.8 \%$ and $67.5 \%$, respectively. These values are consistent with a previously

221 reported 66\% energy recovery for biocrude obtained by contacting DCM directly with the entire

222 post-HTL reaction mixture produced at similar conditions [10].

223 Table 5 Energy recoveries in the water-insoluble $(\mathrm{B})$ and water-soluble $\left(\mathrm{B}_{\mathrm{A}}\right)$ biocrudes and heavy fraction $\left(\mathrm{B}^{\mathrm{H}}\right)$ of

water-insoluble biocrude obtained from $\mathrm{HTL}$ at $350{ }^{\circ} \mathrm{C}$.

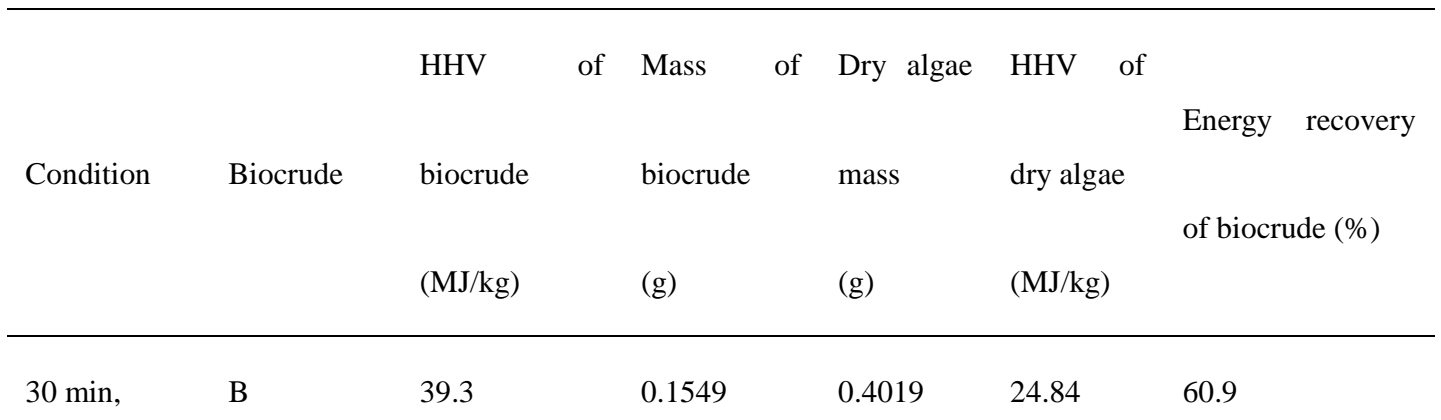




\begin{tabular}{|c|c|c|c|c|c|c|}
\hline $14.1 \quad \mathrm{wt} \%$ & $\mathrm{~B}_{\mathrm{A}}$ & 30.4 & 0.0160 & & & 4.89 \\
\hline loading & $\mathrm{B}^{\mathrm{H}}$ & 35.5 & 0.0600 & & & 21.4 \\
\hline & B & 38.7 & 0.1055 & & & 59.5 \\
\hline & $\mathrm{B}_{\mathrm{A}}$ & 30.4 & 0.0181 & 0.2765 & 24.84 & 8.00 \\
\hline & $\mathrm{B}^{\mathrm{H}}$ & 36.4 & 0.0454 & & & 24.1 \\
\hline
\end{tabular}

\section{3.4. Composition of Biocrude from Aqueous Phase}

226 The biocrude that was extracted from the aqueous phase, $\mathrm{B}_{\mathrm{A}}$, was analyzed by GC-MS. Fig. 2 shows

227 the total ion chromatograms for $\mathrm{B}_{\mathrm{A}}$ obtained at different HTL reaction times. The water-soluble

228 biocrude components included compounds tentatively identified as pyrazines, piperidenones,

229 cyclopentanone, amides, pyrrolidinones and pyrrolidinediones, pyrrolidines, phenols, and amines.

230 There are numerous heterocyclic compounds containing $\mathrm{N}$ and/or $\mathrm{O}$ atoms. Oxygenated compounds can

231 be produced by the degradation of lipids and carbohydrates, whereas protein decomposition could yield

232 numerous nitrogen heterocycles [4]. The presence and prevalence of compounds containing $\mathrm{N}$ and $\mathrm{O}$ are

233 consistent with the elemental analysis results, which showed that the water-soluble biocrude

234 components are richer in $\mathrm{N}$ and $\mathrm{O}$ than is the water-insoluble biocrude. A high $\mathrm{N}$ content in the biocrude

235 negatively affects its combustion and various other properties [2]. Thus, this higher heteroatom content

236 above implies that the biocrude would be of lower quality.

237 a) 

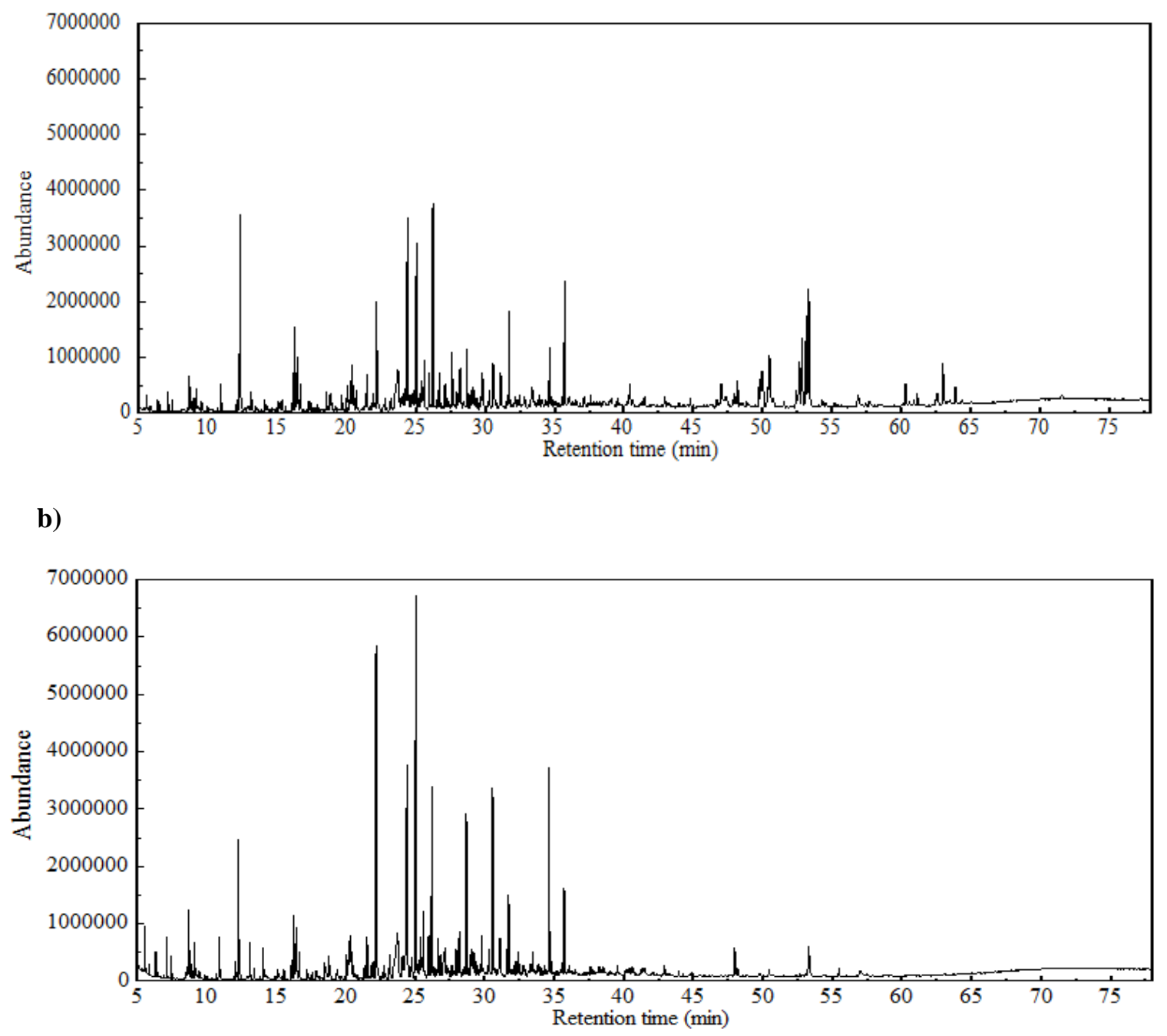

241 c)

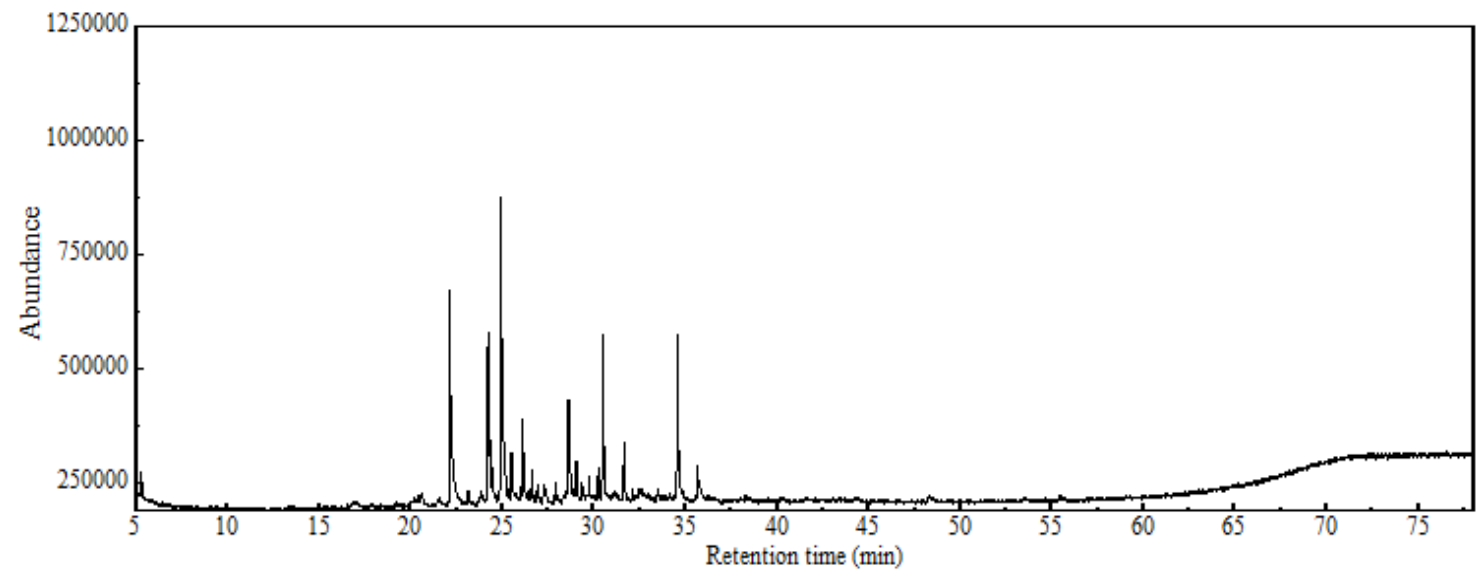

Fig. 2. Total ion chromatograms for water-soluble biocrude obtained from $\mathrm{HTL}$ at $350{ }^{\circ} \mathrm{C}$ and $14.1 \mathrm{wt} \%$ algae loading 
245 As the HTL reaction time increased, the relative amounts of the different compounds in the

246 water-soluble biocrude varied. Compounds with retention times longer than $36 \mathrm{~min}$ are common in the

247 biocrude formed after HTL for $10 \mathrm{~min}$, but largely absent in the biocrude recovered from HTL at longer

248 times. Likewise, the compounds with retention times before 20 min vanish from the chromatogram for

249 the biocrude formed after HTL for $60 \mathrm{~min}$.

\subsection{Functional group compositions}

251 We conducted ${ }^{1} \mathrm{H}$ - and ${ }^{13} \mathrm{C}-\mathrm{NMR}$ analyses of biocrudes to identify the types of structures and

252 functional groups present. Fig. 3 depicts the ${ }^{1} \mathrm{H}$ NMR spectra of the biocrudes obtained from HTL at 30

$253 \mathrm{~min}$ and $350{ }^{\circ} \mathrm{C}$ and their light and heavy fractions. These spectra are representative of the features in the

254 biocrudes produced at other reaction conditions investigated. All of the spectra show resonances at

255 around $0.8,1.2,2.1$, and $7.2 \mathrm{ppm}$, though the relative height of each peak varied from sample to sample.

256 The peaks at 0.8 and 1.2 are characteristic of terminal methyl groups and methylene groups in alkyl

257 chains, respectively [11]. The peak at about $2.1 \mathrm{ppm}$ is consistent with the resonance expected from

258 protons on carbon atoms $\alpha$ to a carbonyl group. The peaks near $7.2 \mathrm{ppm}$ likely arose from aromatic

259 protons or conjugated dienes [12,13]. The appearance of peaks at these resonances reveals the presence

260 of alkyl moieties, carbonyl functionalities, and aromatic or unsaturated molecules in all of the biocrudes,

261 though in differring amounts. Our previous article discussed the differences between the NMR spectra

262 for the biocrudes that separated spontaneously from the aqueous phase and that was extracted from the

263 aqeuous phase, so we focus on a comparison of the light and heavy fractions here. To the best of our

264 knowledge, this is the first report of spectroscopic characterization of light and heavy fractions of 
biocrude.

266 Comparing the spectra for the light and heavy biocrudes in Fig $3 \mathrm{c}$ and 3d, one observes peaks around

2671.5 and $1.9 \mathrm{ppm}$ in the light biocrude that are not present in the spectrum for the heavy portion of the

268 same biocrude. Resonances at these locations are indicative of protons on tertiary aliphatic carbon

269 atoms and on allylic carbons. Apparently, there is a greater abundance of such structures in the light

270 fraction of the biocrude than the heavy fraction.

271 Comparing the spectra for the light and heavy components of the biocrude extracted from the aqueous

272 phase (panels e and f) shows that the light fraction has peaks at 2.7 and $2.9 \mathrm{ppm}$ that are absent in the

273 heavy fraction. Resonances from protons that are $\alpha$ to a heteroatom or unsaturated moiety (e.g., benzylic

274 or allylic protons) appear in this region.

275 a)

b)
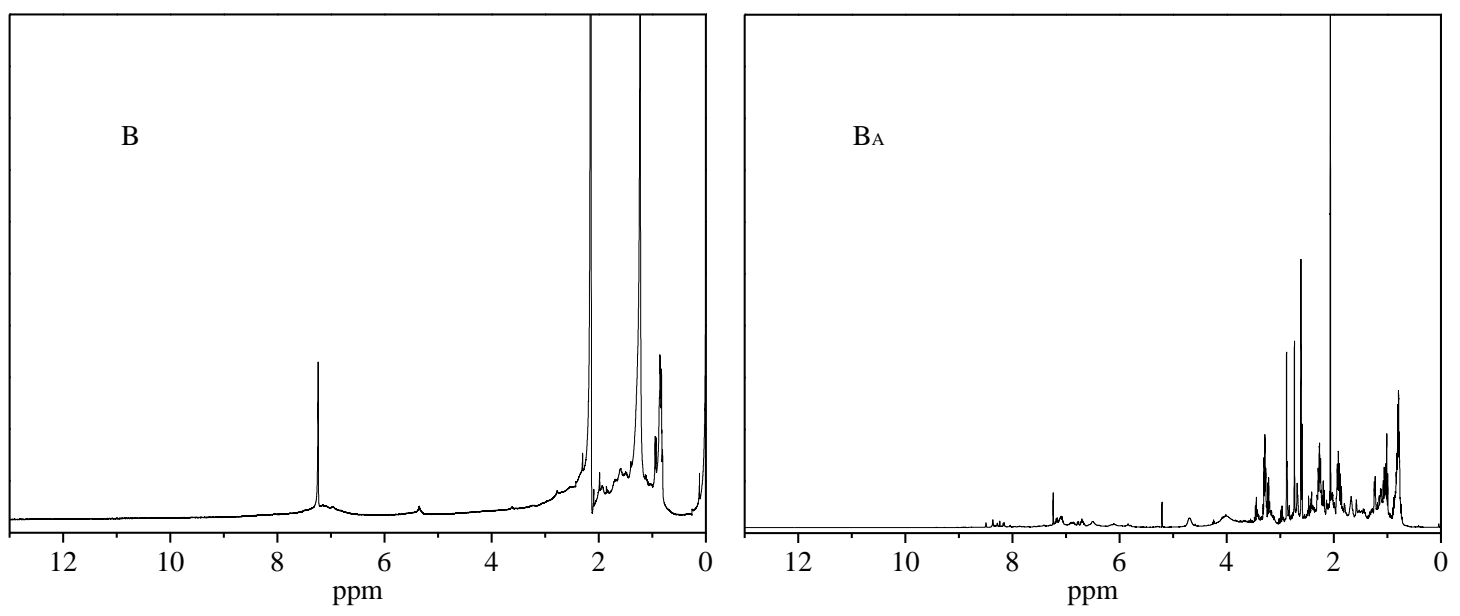

276

277

278

279

c)

d) 

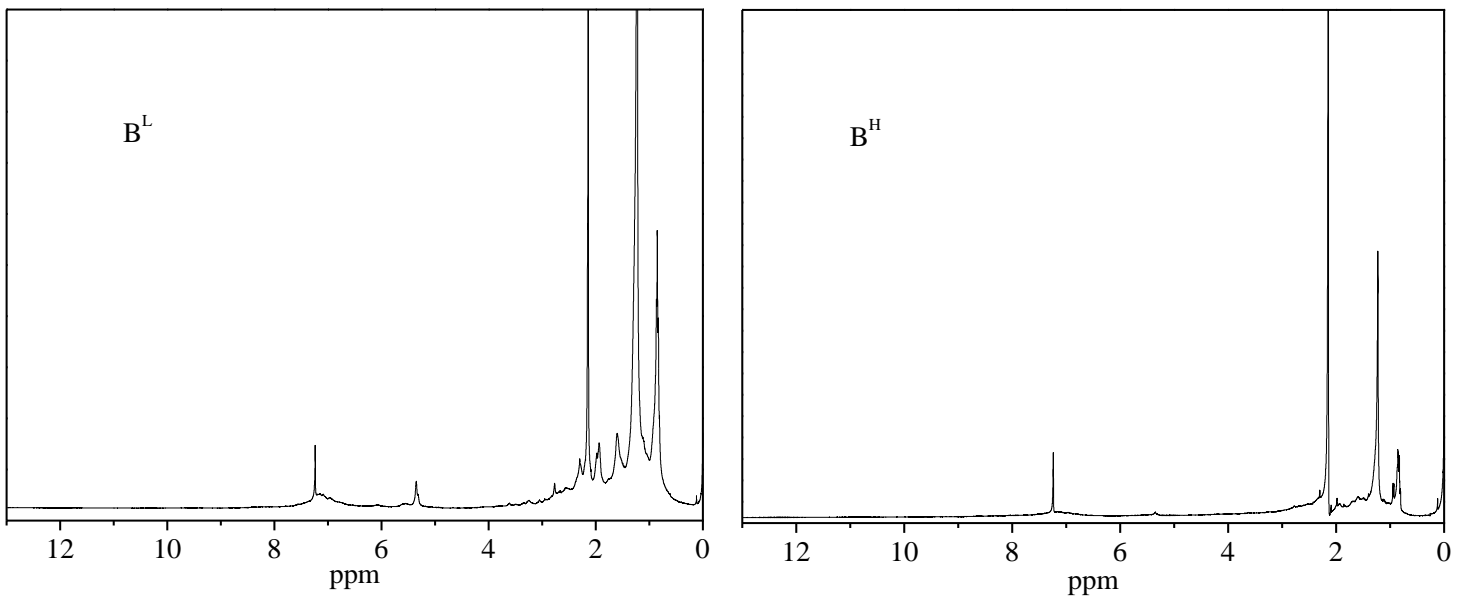

281

e)

f)
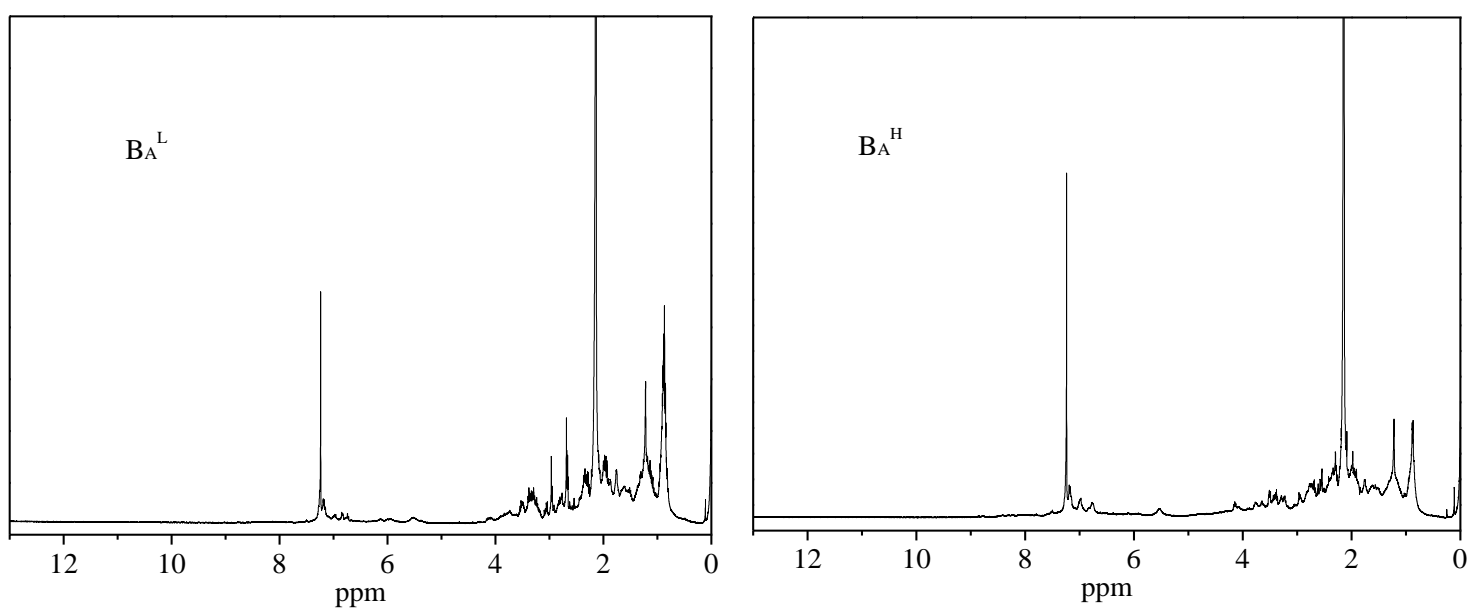

Fig. 3. ${ }^{1} \mathrm{H}-\mathrm{NMR}$ spectra of biocrudes obtained from $\mathrm{HTL}$ at $350{ }^{\circ} \mathrm{C}, 30 \mathrm{~min}$, and $14.1 \mathrm{wt} \%$ algae loading: a)

water-insoluble biocrude $(B), b)$ water-soluble biocrude $\left(B_{A}\right)$, c) light fraction of water-insoluble biocrude $B^{L}$, d) heavy

fraction of water-insoluble biocrude $\mathrm{B}^{\mathrm{H}}$, e) light fraction of water-soluble biocrude $\mathrm{B}_{\mathrm{A}}{ }^{\mathrm{L}}$, and $\mathrm{f}$ ) heavy fraction of

water-soluble biocrude $\mathrm{B}_{\mathrm{A}}{ }^{\mathrm{H}}$.

Fig. 4 displays ${ }^{13} \mathrm{C}$-NMR spectra of the biocrudes and their light and heavy subfractions from HTL at one set of reaction conditions. These spectra are representative of those obtained at other conditions, as the reaction time and algae loading did not influence the appearance of the spectra. Both the water-insoluble and the water-soluble biocrude samples display several peaks in the 0-55 ppm region, 
291 where aliphatic carbon atoms appear [14]. This result is consistent with the high content of aliphatic

292 carbons indicated by the ${ }^{1} \mathrm{H}-\mathrm{NMR}$ spectra $(0.5-3.0 \mathrm{ppm})$. The weak peaks at around $130 \mathrm{ppm}$ are

293 consistent with the presence of alkenyl and aromatic carbon in these biocrudes. Furthermore, all

294 biocrudes in Fig. 3 showed a resonance around 207 ppm, which is in the region (180-215 ppm)

295 representing carbonyl carbons in ketones/aldehydes [15].

296 The spectra for the light and heavy fractions of the water-insoluble biocrude have several peaks in

297 common, but the light fraction has additional peaks in the region from $0-30 \mathrm{ppm}$, suggesting that it

298 contained a larger variety of compounds with methyl and methylene groups. The spectrum for the

299 water-soluble biocrude showed resonances within the region of 165-180 ppm, where carbon atoms in

300 carboxylic acid groups or esters appear [12,15]. The peaks at about $115 \mathrm{ppm}$ and $141-146 \mathrm{ppm}$ are in the

region (95-165 ppm) where aromatic and heteroaromatic carbons appear [15].

a)

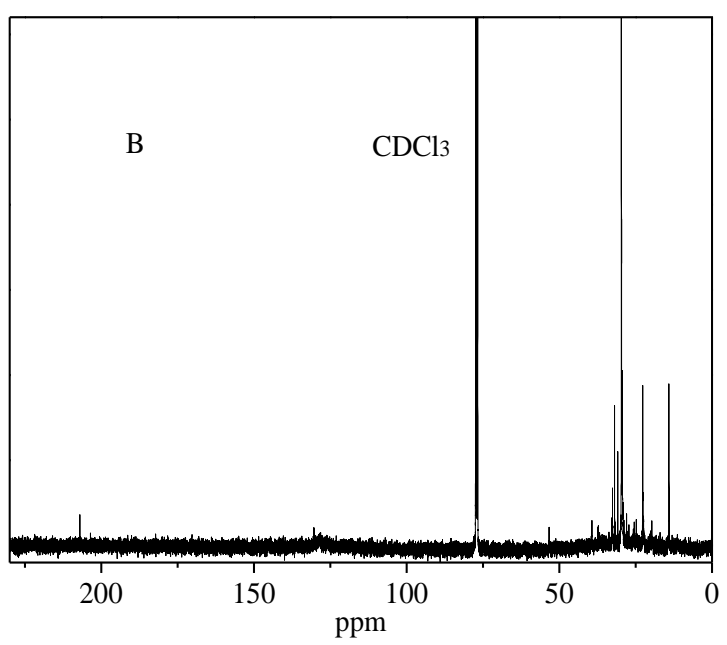

b)

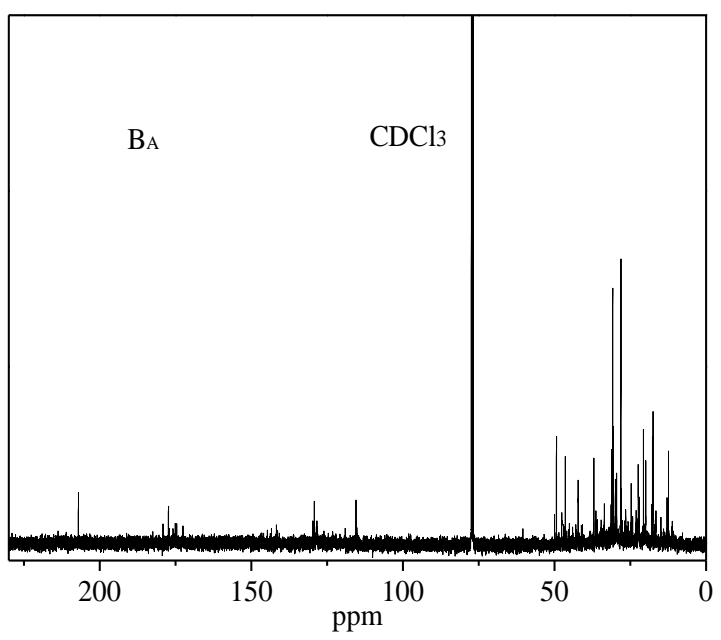



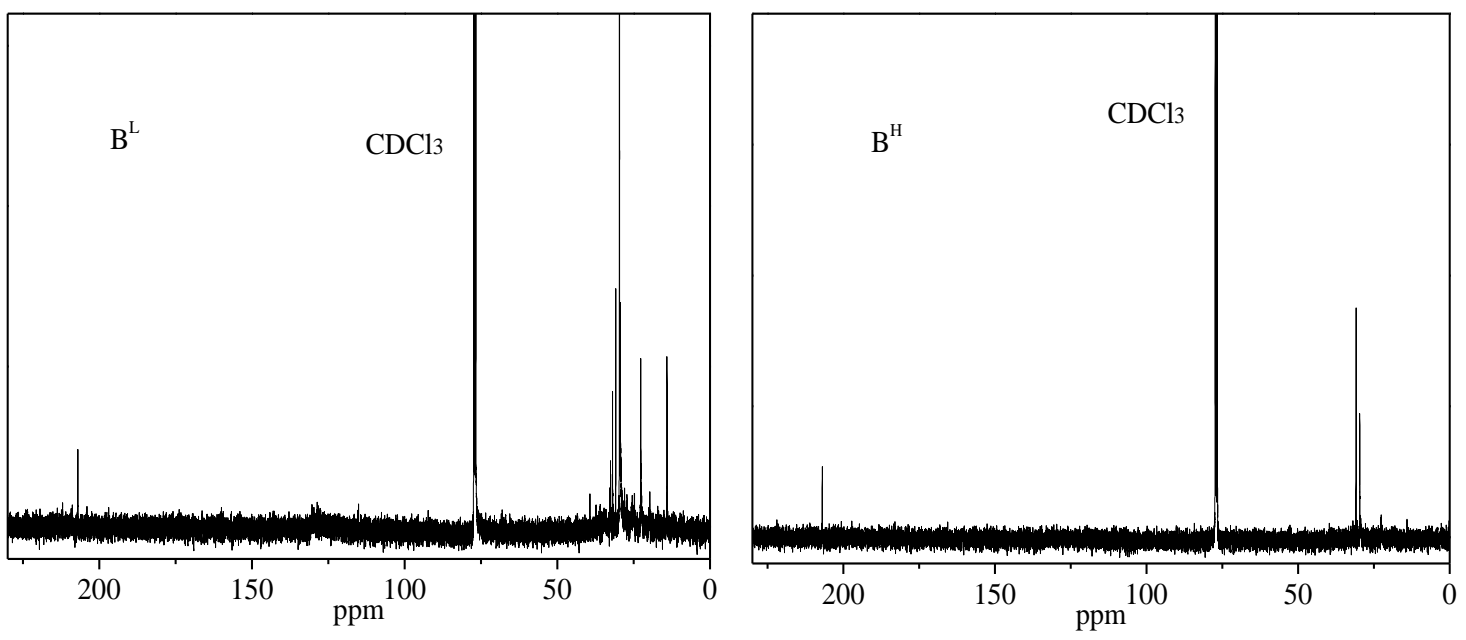

Fig. 4. ${ }^{13} \mathrm{C}-\mathrm{NMR}$ spectra of biocrudes obtained from $\mathrm{HTL}$ at $350{ }^{\circ} \mathrm{C}, 30 \mathrm{~min}$, and $14.1 \mathrm{wt} \%$ algae loading. a) heavy fraction of water-insoluble biocrude $\mathrm{B}^{\mathrm{H}}$.

\section{Conclusions} co-product declined from $6.4 \mathrm{wt} \%$ to $3.6 \mathrm{wt} \%$ but the yield of biocrude that spontaneously separated from the aqueous phase after cooling the HTL products increased from $38.2 \mathrm{wt} \%$ to $40.5 \mathrm{wt} \%$. Algae loading had a much stronger influence. As the wt $\%$ of algae in the slurry feedstock increased, the yield of water-soluble biocrude decreased to $2.55 \mathrm{wt} \%$, but the yield of water-insoluble biocrude increased by nearly $50 \%$. Longer reaction times and higher algae loadings favor reactions that produce molecules that are insoluble in ambient liquid water. Reactions such as decarboxylation, deamination, dehydration, oligomerization, and condensation could be responsible for this shift in the product distribution. The 
322 heating value of the biocrudes showed little variation with HTL processing time, but the heating values

323 of the biocrudes produced at the higher algae loadings always exceeded those of the biocrudes produced

324 at the lower loadings. This trend held for both the water-soluble and water-insoluble biocrude

325 components. The results presented herein demonstrate that the HTL reaction time and the wt $\%$ algae in

326 the feedstock affect the relative amounts of water-soluble and water-insoluble biocrude compounds and

327 the heating value of the biocrudes produced.

328 Acknowledgements

329 We gratefully acknowledge financial support from the University of Michigan College of 330 Engineering, the National Science Foundation (EFRI-0937992), the National Natural Science 331 Foundation of China (No.21206132), the Specialized Research Fund for the Doctoral Program of 332 Higher Education (No.20120201120069), the Fundamental Research Funds for the Central Universities 333 (No. xjj2012032), the National Science Foundation for Post-doctoral Scientists of China (No. 334 2013M540748), and the seventh batch of special fund of the National Science Foundation for 335 Post-doctoral Scientists of China (No.126568) and the State Scholarship Fund for University Key 336 Teachers from the Ministry of Education of China to Study Abroad as a Visiting Scholar (Grant No. $337201206285008)$.

\section{$338 \quad$ References}

339 [1] A.A. Peterson, F. Vogel, R.P. Lachance, M. Fröling, M.J. Antal Jr, J.W. Tester, Thermochemical biofuel production 
341 [2] S.S. Toor, L. Rosendahl, A. Rudolf, Hydrothermal liquefaction of biomass: A review of subcritical water

342 technologies, Energy 36 (2011) 2328-2342.

343 [3] D. López Barreiro, W. Prins, F. Ronsse, W. Brilman, Hydrothermal liquefaction (HTL) of microalgae for biofuel

344 production: State of the art review and future prospects, Biomass Bioenergy 53 (2013) 113-127.

345 [4] N. Sudasinghe, B. Dungan, P. Lammers, K. Albrecht, D. Elliott, R. Hallen, T. Schaub, High resolution FT-ICR mass

346 spectral analysis of bio-oil and residual water soluble organics produced by hydrothermal liquefaction of the marine microalga Nannochloropsis salina, Fuel 119 (2014) 47-56.

[5] D.C. Elliott, T.R. Hart, G.G. Neuenschwander, L.J. Rotness, G. Roesijadi, A.H. Zacher, J.K. Magnuson, $207-215$.

[6] D.C. Elliott, T.R. Hart, A.J. Schmidt, G.G. Neuenschwander, L.J. Rotness, M.V. Olarte, A.H. Zacher, K.O. Albrecht, reactor, Algal Res. 2 (2013) 445-454.

[7] P.J. Valdez, J.G. Dickinson, P.E. Savage, Characterization of product fractions from hydrothermal liquefaction of nannochloropsis sp. and the influence of solvents. Energy Fuels 25 (2011) 3235-3243.

[8] D.H. Xu, P.E. Savage, Characterization of biocrudes recovered with and without solvent after hydrothermal liquefaction of algae, Algal Res. 6 (2014) 1-7.

[9] M. Biddy, R. Davis, S. Jones, Y. Zhu, Whole Algae Hydrothermal liquefaction technology pathway, technical report 
363 New York, 2005.

364 [12] P.G. Duan, P.E. Savage, Hydrothermal liquefaction of a microalga with heterogeneous catalysts, Ind. Eng. Chem.

365 Res. 50 (2010) 52-61.

366 [13] C.A. Mullen, A.A. Boateng, Characterization of water insoluble solids isolated from various biomass fast pyrolysis

367 oils, J. Anal. Appl. Pyrol. 90 (2011) 197-203.

368 [14] P.G. Duan, P.E. Savage, Upgrading of crude algal bio-oil in supercritical water, Bioresour. Technol. 102 (2011)

1899-1906.

370 [15] G.D.S. C.A. Mullen, A.A. Boateng, Characterization of various fast-pyrolysis bio-oils by NMR spectroscopy,

371 Energy Fuels 23 (2009) 2707-2718. 